\title{
Optimized Operation of Photonic Devices With Use of Ordinary Bulk Materials
}

\author{
Constantinos Valagiannopoulos ${ }^{1}$ \\ ${ }^{1}$ Department of Physics, School of Science and Technology, Nazarbayev University, \\ Qabanbay Batyr Ave 53, 010000, Astana, Kazakhstan \\ konstantinos.valagiannopoulos@nu.edu.kz
}

\begin{abstract}
Structural boundaries, materials and feeding sources are the three fundamental segments defining photonic devices. Since excitation is usually dictated by the application and there are infinite ways to select the spatial configuration of the component, an optimization with respect to the used media is both doable and useful. We provide several combinations of elements and compounds making high-performing electromagnetic devices in terms of absorption, scattering and unusual refraction with simple structures like bilayers, two- and three- dimensional core-shell particles or binary metasurfaces. Such large sets of potential candidates for the employed media can be deployed by experimentalists after applying a secondary sweep by imposing additional constraints concerning ease of fabrication.
\end{abstract}

\section{INTRODUCTION}

Practically any electromagnetic device is a combination of three ingredients shown in the abstract schematic of Fig. 1(a). First of all, one should sketch the shape of the boundaries (structure), defining the volumes of the separate components of the device. To this end, the designer must decide the materials with which the aforementioned volumes will be filled and finally the spatial and time profile of the sources that will feed the photonic system. By solving such a structure, we mean determining the unique spatio-temporal distribution of the electromagnetic fields. It becomes feasible by considering the forms of the general solution to the Helmholtz equation into each volume (dependent on the material) and imposing the boundary conditions across the interfaces of each pair of adjacent volumes (dependent on the shape of the boundaries). Once a primary source is present, the obtained set of equations has a non-zero inhomogeneous part and thus the unique output of the device is deduced (direct solving in Fig. 1(a)). If no primary source exists, then we can only find the waveforms of the fields across the device's geometrical extent instead of the exact values of them (waveguides or resonators).

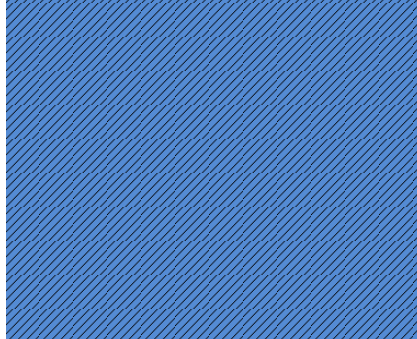

(a)

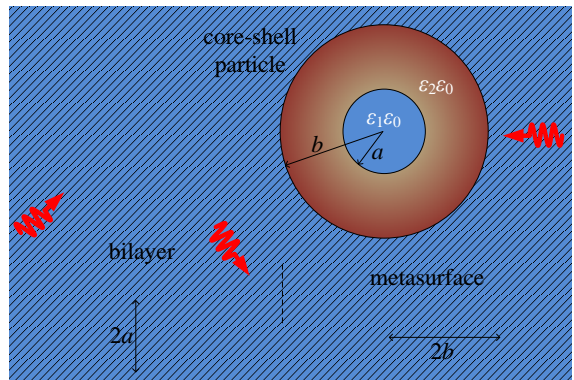

(b)

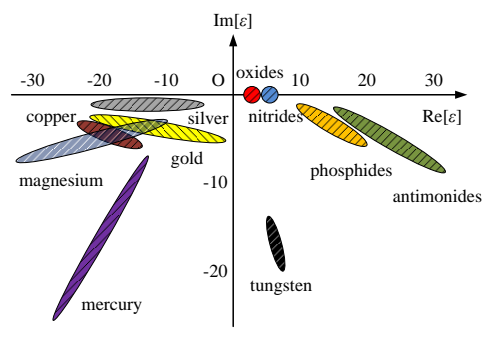

(c)

Fig. 1: (a) Schematic diagram of forward and inverse design of an electromagnetic system. (b) Simple photonic structures (bilayer, core-shell nanoparticles, grating metasurfaces). (c) Approximate loci of the complex permittivities of basic metals and chemical compounds for various frequencies of the visible light.

As long as one has procured the spatial distribution of light into every single volume of our system, one should find the correct metric in order to evaluate the performance of the device. Obviously, different operations make 
different metrics; however, even for a structure that has been designed for a specific aim, there can be several score indicators, less or more successful. The success in selecting a metric is rated by how fast and efficiently one can perform reverse engineering to find the optimal design that maximizes itself (inverse design in Fig. 1(a)). Given the fact that there are countless different ways to pick the structural configuration of the device and the source is usually given by the application, the only system's ingredient that remains to be optimized in a tractable and systematic way, are the used media. Indeed, in any ordinary nano-fabrication laboratory, only a restricted number of chemical elements and compounds is available to construct a stable design.

This is the objective of the present work: we consider various simple structures (bilayers, core-shell nanoparticles or gradient metasurfaces) under plane-wave illumination as presented in Fig. 1(b). We examine what are the combinations of materials giving high performances in terms of various objectives like absorption, scattering and anomalous refraction. In this way, a useful toolbox is provided to the interested experimental scientist wilth multiple alternative designs serving the prescribed purpose. We do mainly care about operational frequencies of the visible spectrum where constituent properties of metals and semi-conductors exhibit meaningful variations as indicated by the approximate loci of the complex permittivity plane $(\operatorname{Re}[\varepsilon], \operatorname{Im}[\varepsilon])$ in Fig. 1(c), according to [1].

\section{MAIN BODY}

Since our intention is to stress the significance of using the correct raw materials to maximize the performance of a specific class of photonic devices, we select certain very simple configurations and excitations (TE/TM plane wave). In particular, bilayers are comprised of two slabs connected back-to-back and make the simplest possible Cartesian design supporting electromagnetic resonances which give rise to interesting effects such as field enhancement [2] and wide-angle absorption [3]. As far as non-planar geometries are concerned, we are considering core-shell nanowires [4] and nanospheres [5] which can be also self-assembled [6]. Finally, we regard binary metasurfaces [7] with rectangular or circular cylinders playing the role of their constituent elements (meta-atoms) . All these structures are tested with respect to their performance in terms of various operations including absorption, scattering and unusual refraction [8].

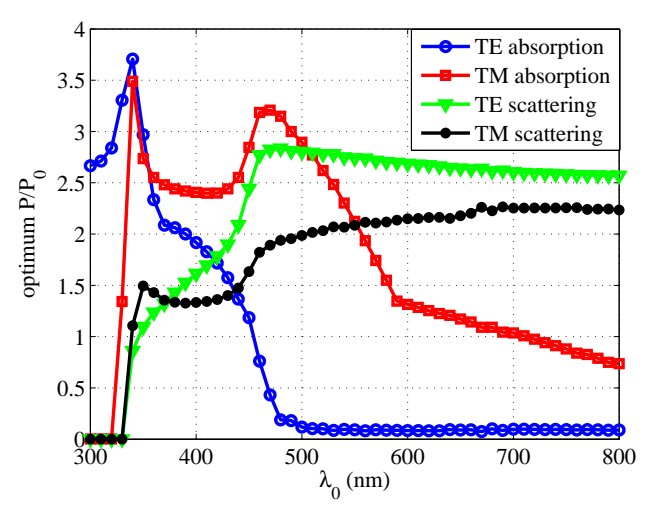

(a)

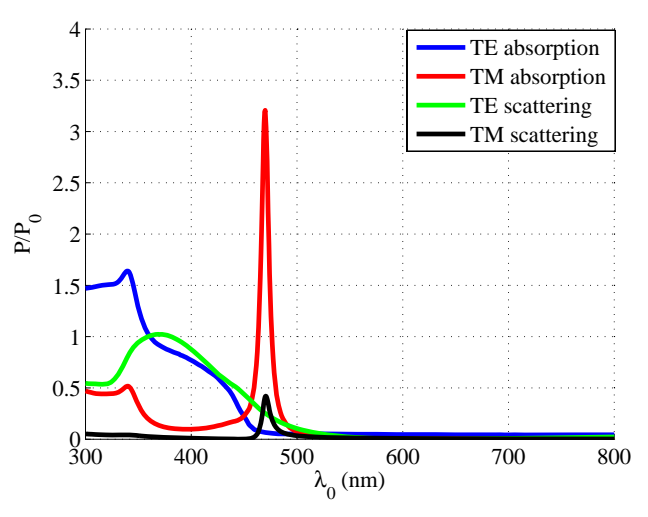

(b)

Fig. 2: (a) The optimal absorbed and scattering powers of $\mathrm{Ag} / \mathrm{GaP}$ core-shell nanoparticles for both polarizations, normalized by suitable quantities as functions of free-space wavelength $\lambda_{0}$. (b) The absorbed and scattering powers of a specific $\mathrm{Ag} / \mathrm{GaP}$ core-shell nanoparticle which works optimally for the absorbing operation of TM polarization at $\lambda_{0}=470 \mathrm{~nm}$, as functions of free-space wavelength $\lambda_{0}$.

We have tried every combination of typical materials employed in nano-fabrication (some of which are mentioned in Fig. 1(c)) and for every single of them we have found the geometrical characteristics giving maximal performance with respect to various operational aims described above. In this way, certain "rules of thumb" related to the preferences of each material for certain frequencies can be obtained as in [3] for the bilayer case. In Fig. 2(a), we show the maximum absorbing $P_{a b s}$ and scattering $P_{\text {scat }}$ powers of core-shell nanowires (with silver core and Gallium Phosphide shell) under plane-wave excitation for both polarizations (TE/TM) and as functions of the free-space wavelength $\lambda_{0}$. The absorbing power is normalized by the power of the incident plane wave passing 
through the geometrical cross section of the particle if it is conditionally removed $P_{0, a b s}$ and the scattering power is normalized by the corresponding quantity $P_{0, \text { scat }}$ if the particle was a suitable perfect conductor (PEC/PMC). We search for maximal performance in the case of small particles with diameter less than half wavelength $\lambda_{0} / 2$ and we reject the optima appeared at the endpoints of our variables' intervals. If no strict maximum is observed within the considered parametric space, we assign to the represented quantity a negligible value. Every single point of the four different curves depicted in Fig. 2(a) corresponds to a different optimal particle. If we pick one of them, namely the one that maximally absorbs the TM illumination at $\lambda_{0}=470 \mathrm{~nm}$ (blue light), we obtain the responses shown in Fig. 2(b). Naturally at the optimal frequency $\lambda_{0}=470 \mathrm{~nm}$, TM absorption takes huge values, while at the rest of the wavelengths $\lambda_{0}$ the performances are (much or less) inferior to the corresponding maximal ones of Fig. 2(a).

\section{Conclusions}

Various optimally absorbing, scattering and refraction configurations are determined with use of ordinary metals, plasmonics, semiconductors and dielectrics in the visible. The aforementioned results are indicative and concern only one type of photonic structures from Fig. 1(b) (core-shell nanocylinders). More results are being prepared and will be presented at the conference. They unveil interesting correlations between the used media and each operation, oscillation wavelengths and type of excitation.

\section{ACKNOWLEDGEMENT}

This work was partially supported by Nazarbayev University Small Grants with project entitled: "Super transmitters, radiators and lenses via photonic synthetic matter” (No. 17095) and Nazarbayev University ORAU Grant entitled: "Structured light for nonlinear and topological photonics" (No. 20162031). Funding from MES RK state-targeted program BR05236454 is also acknowledged.

\section{REFERENCES}

[1] M. N. Polyanskiy, Refractive Index Database, http://refractiveindex.info. Accessed on 2018-01-30.

[2] S. Savoia, G. Castaldi, V. Galdi, A. Alu, and N. Engheta, "Tunneling of obliquely incident waves through PT-symmetric epsilon-near-zero bilayers," Physical Review B, vol. 91, 2014.

[3] A. N. Papadimopoulos, N. V. Kantartzis, N. L. Tsitsas, and C. A. Valagiannopoulos, "Wide-angle absorption of visible light from simple bilayers," Applied Optics, vol. 56, 2017.

[4] L. Cao, J. S. White, J.-S. Park, J. A. Schuller, B. M. Clemens, and M. L. Brongersma, "Engineering light absorption in semiconductor nanowire devices," Nature Materials, vol. 8, 2009.

[5] D. C. Tzarouchis and A. Sihvola, "General scattering characteristics of resonant coreshell spheres," IEEE Transactions on Antennas and Propagation, vol. 66, 2018.

[6] Z. Nie, A. Petukhova, E. Kumacheva, "Properties and emerging applications of self-assembled structures made from inorganic nanoparticles," Nature Nanotechnology, vol. 5, 2010.

[7] F. Ding, A. Pors and S. I. Bozhevolnyi, "Gradient metasurfaces: a review of fundamentals and applications," Reports on Progress in Physics, vol. 5, 2010.

[8] N. L. Tsitsas and C. A. Valagiannopoulos, "Anomalous reflection of visible light by all-dielectric gradient metasurfaces," Journal of Optical Society of America B, vol. 34, 2017. 